\title{
Nickel Nanoparticles Immobilized on a Porous Triazine-Thiourea-Sulfonamide as an Efficient Heterogeneous Catalyst for Reduction of Carbonyl Compounds
}

\author{
Abumuslim Rahimi \\ Bu-Ali Sina University \\ Ramin Ghorbani-Vaghei ( $\sim$ ghorbani@basu.ac.ir ) \\ Bu-Ali Sina University \\ Sedigheh Alavinia \\ Bu-Ali Sina University
}

\section{Research Article}

Keywords: triazine-thiourea-sulfonamide (TTSA), mesoporous catalyst, aldehydes, ketones, formic acid/triethyl amine

Posted Date: January 23rd, 2021

DOI: https://doi.org/10.21203/rs.3.rs-148884/v1

License: (c) (1) This work is licensed under a Creative Commons Attribution 4.0 International License.

Read Full License 


\section{Abstract}

In order to investigate the role of silica template, polymers and nickel nanoparticles on the reduction of aldehydes/ketones, a novel porous triazine-thiourea-sulfonamide polymeric organic support (TTSA) was prepared via in-situ polymerization of melamine (1,3,5-triazine-2,4,6-triamine), thiourea and benzene-1,3disulfonyl chloride in the presence of silica nanoparticles as a template led to the synthesize silica TTSA nanocomposite. Next, after removal of the template, the nanocomposite was transformed into mesoporous poly triazine-thiourea-sulfonamide. Ni nanoparticles (Ni NPs) were then decorated on the designed mesoporous polymer support and the resulting TTSA@Ni NPs nanospheres were employed as heterogeneous catalyst in the construction of alchohols from reduction of aldehydes/ketones using formic acid/triethyl amine as a reducing agent in water as solvent. The catalyst is low-cost, non-toxic, and can be reused for several catalytic cycles without decreasing the activity.

\section{Introduction}

Recently, great emphasis is given to the role of environment-friendly technologies in chemical reactions. In relation to environment-friendly technologies, catalytic processes (e.g. toxic and expensive reagents removal, minimization of by-products, and simplifying of workup procedures) are particularly important factors influencing the efficiency of the organic reaction ${ }^{1}$. Among the significant novel synthetic protocols developed in organic chemistry, the selective reduction of aldehydes and ketones to alchohol is most interesting one ${ }^{2}$. Alcohols can be converted to the various valuable chemicals using etherification, condensation, esterification, and oxidation ${ }^{3-5}$. In this regard, the importance of alcohol production will enforce the development of novel technologies for the production of alcohols using nanocomposites.

Recently, synthesis of metallic nanoparticles (M NPs) for use as catalyst have gained particular interest in different organic reactions ${ }^{6-9}$. The nano shape, size and a large surface area by volume ratio provides unique characterstics to nanocatalysts due to the structural and electronic changes which differentiates them from the original material ${ }^{10}$. However, the self-agglomeration and leaching problems can be negatively effect on catalytic or recycling performance of M NPs ${ }^{11-12}$. Selection or preparation of ideal catalyst supports, which have high surface area and ligation sites to strongly interact with M NPs, can be regarded as an efficient way to minimize these problems ${ }^{13}$. Up to now, different organic or inorganic solid catalyst supports have used to immobilize various M NPs ${ }^{14-16}$.

In this regard, porous cross-linked polymers consisting of different functional group, used as powerful heterogeneous catalysts used to generate several novel organic reactions under mild conditions, providing high selectivity. Their ease of handling and processing, recyclability, high thermal and chemical stabilities, low-cost production and large surface areas are important factors influencing the efficiency of the catalyst application of porous cross-linked polymers ${ }^{17}$. Although different methods have been reported for the creation of porousity in polymers (self-assembly, block copolymer, template techniques, and a combination of templating and self-assembly techniques), in fact, silica template technique has 
gained a great attention in catalysis as it improves the efficiency of catalyst by high metal loading, high surface area and pore volume which may favorable for easy sorption of reactant and products ${ }^{18-20}$.

To date, bifunctional organocatalysts which contains sulfonamide and a basic amine moiety have attracted remarkable attention of organic chemists due to its numerous synthetic applications ${ }^{21}$. Polysulfonamides are synthesized by the reaction of disulfonyl chloride and various amines. Cross-linked polysulfonamides, which are novel category of polysulfonamides, has the ability to immobilize different MNPs $^{22}$. Cross-link is a bond which links one polymer chain to another. In this study, porous cross-linked triazine-thiourea-sulfonamide (TTSA) is considered as a novel support which has the ability to immobilize $\mathrm{Ni}$ nanoparticles. Porous TTSA was prepared from insitu polymerization of benzene-1,3-disulfonyl chloride, thiourea and melamine and silica nanoparticles. Melamine was used as a cross-linker and silica was used as a template. In the second step, removal of the silica template led to the synthesis of porous triazine-thiourea-sulfonamide (TTSA). Finally, porous TTSA was used as a mesoporous substrate for the stabilization of Ni nanoparticles (Scheme 1). The presence of sulfonamide, thiourea and melamine groups lead to facile immobilization of Ni NPs with high loading capacity, high chemical and thermal stability. Thus, the development of multifunctional polysulfonamide bearing sulfonamide, melamine and thiourea groups along with hydrophilicity and high surface area, as a novel porous support, can revolutionize the polysulfonamide chemistry.

In continuation of our interest to design of polysulfonamide-based catalyst for different organic reactions ${ }^{23}$, herein, we synthesized a novel stabilizer composed of triazine-thiourea-sulfonamide (TTSA) as a novel category of polysulfonamides. Nickel nanoparticles were then successfully immobilized on prepared support (Scheme 1) and fully characterized by FT-IR, FE-SEM, EDS, TGA, $\mathrm{N}_{2}$-adsorptiondesorbtion and WDX. Next, catalytic role of TTSA@Ni nanocomposite were studied in the green synthesis of alchohols from the reduction of aldehydes and ketones. The presence of thiourea and melamine as chelating groups plays an important role in the immobilization of Ni NPs. Moreover, the amphiphilicity property of polymer substrate, which is due to the presence of hydrophilic monomers (thiourea and melamine), lead to the green reduction of aldehydes/ketones in aqueous solution. Additionally, we have tested recyclability/reusability of TTSA@Ni nanocomposite (Scheme 2). Catalytic studies indicate that TTSA@Ni nanocomposite has significant catalytic performance by producing various alchohols with high reaction yields. More importantly, used TTSA@Ni nanocomposite easily recollected via centifusion and were reused several times in consecutive reactions by negligible leaching.

\section{Result And Discussion}

\section{Characterization of TTSA@Ni NPs}

The FT-IR spectrum of $\mathrm{SiO}_{2} @$ TTSA (A), porous TTSA (B), TTSA@Ni (C), are shown in Fig. 1. Initially, FT-IR analysis of $\mathrm{SiO}_{2} @$ TTSA displayed obvious peaks at $1172 \mathrm{~cm}^{-1}$ and $1462 \mathrm{~cm}^{-1}$ are related to the Si-O stretching vibrations of silica nanoparticles. The broadening peak at $3200-3400 \mathrm{~cm}^{-1}$ confirmed the 
presence of the $\mathrm{N}-\mathrm{H}$ and $\mathrm{NH}_{2}$ bond. In the FT-IR of $\mathrm{SiO}_{2} @ T T S A$, the stretching vibration at 1184 and 1411 $\mathrm{cm}^{-1}$ for the $\mathrm{SO}_{2}$ bands, indicating the presence of sulfonamide groups. In addition, the adsorption peak at $1716 \mathrm{~cm}^{-1}$ belong to the $\mathrm{C}=\mathrm{S}$ band in the thiourea groups. The peaks at $1668 \mathrm{~cm}^{-1}$ approved the presence of melamine in the structure of $\mathrm{SiO}_{2} @$ OTTSA nanocomposite (Fig. 1A). There is no significant change in the position of peaks, after silica etching (Fig. 1B). In the final step (Fig. 1C), the bending vibration absorption peak of $\mathrm{C}=\mathrm{N}$ shifted to $1640 \mathrm{~cm}^{-1}$, that this shift confirms the Ni NPs coordination of the $\mathrm{C}=\mathrm{N}$ of melamine groups.

The size and morphology of porous substrate (TTSA) and the prepared catalyst TTSA@Ni were investigated using FE-SEM, which was shown the mesoporous structure of TTSA (Fig. 2A). FE-SEM image of TTSA@Ni demonstrated spherical shape morphology and uniform distribution of Ni NPs on porous polymer matrix (Fig. 2B).

The EDS analysis of the TTSA@Ni NPs nanocatalyst reveals the signals of The Ni, O, S, C and N (Fig. 3). Elemental mapping for the prepared catalyst exhibits the homogeneous distribution of the elements $(\mathrm{C}, \mathrm{N}$, $\mathrm{Ni}, \mathrm{O}$, and $\mathrm{S}$ ) in the structure of the prepared catalyst as represented in the Fig. 4 . The element Ni content in the TTSA@Ni NPs catalyst was confirmed to be $1.794 \mathrm{mmol} \mathrm{g}^{-1}$, which was recorded by Inductively Coupled Plasma Mass Spectrometry.

TGA curve of TTSA@Ni NPs shown in Fig. 5, illustrated a small lose weight at $100{ }^{\circ} \mathrm{C}$, which could be related to the physically absorbed water and organic solvents. The mass loss at higher temperatures $\left(200-600{ }^{\circ} \mathrm{C}\right)$ can be correlated to the decomposition of organic group of the polymeric structure (Fig. 5).

The Brunauer-Emmett-Teller (BET) surface areas were determined by $\mathrm{N}_{2}$ adsorption (Fig. 6), the surface areas for porous TTSA was found to be $22.23 \mathrm{~m}^{2} / \mathrm{g}$ and total pore volume is $0.07 \mathrm{~cm}^{3} \mathrm{~g}^{-1}$. The porosity of TTSA is important to adsorb the Ni NPs in it. These important surface properties of TTSA can strongly help to the present active catalytic sites for selective hydration of carbonyl compounds into corresponding benzylalchohols.

In order to investigate the amphiphilicity of porous TTSA@Ni, contact angle measurements were applied for water and oil droplets (Fig. 7.). Beacause the contact angle between TTSA@Ni and water (Fig. 7 A) or TTSA@Ni and oil (Fig. 7. B) were below 90', the good amphiphilicity was observed in the reaction medium.

\section{Catalytic studies}

The effect of various reaction parameters, including the amount of catalyst, solvents, hydrogen doner agent, and temperature were investigated in the transfer hydrogenation of benzaldehyde which was selected as test substrate (Table 1). As Table 1 shows, the model reaction gave negligible yields in the absence of any catalyst at room temperature (entry 1 ). As a starting point, the effect of different hydrogen doner including formic acid, ammonium format, formic acid/ammonium format and formic 
acid/triethyl amine was investigated on the model reaction in the presence of $0.03 \mathrm{~g}(5.4 \mathrm{~mol} \%)$ TTSA@Ni as the catalyst. Formic acid and ammonium format were found ineffective system (entry no. 2 and 3 ). Then, the effect of formic acid/ammonium format was investigated in various $\mathrm{pH}$ including 0.5 , $1.55,2,3,4,4.5$, and 5 . Notably, pH plays crucial role in the reaction and the superior activity of the TTSA@Ni was vividly observed in pH of 2.5 (entry 4).Then, the catalytic activity of TTSA@Ni was investigated in the presence of $\mathrm{HCOOH} / \mathrm{NEt}_{3}$ in various $\mathrm{pH}$ (entries 5-10). As seen in Table 1, replacement of formic acid/ammonium format with formic acid/triethyl amine in $\mathrm{pH}$ of 3.2 significantly increases the yield (entry 8). It should be noted, that decreasing the temperature has a negative effect on the progress of the model reaction (entry 11). Furthermore, the model reaction was then performed in the presence of different solvents (entries 12-15) and solvent-free condition (entry 16), and $\mathrm{H}_{2} \mathrm{O}$ provided the desired product in the best yield (entry 8). Finally, the model reaction was examined in the presence of $0.05 \mathrm{~g}(9$ mol\%) TTSA@Ni, initially yield \% is increased with increasing TTSA@Ni up to $0.03 \mathrm{~g}$ (5.4 mol\%) but afterward reaction was found independent to amount of catalyst (entry 17).

To further examine the applicable of TTSA@Ni catalyst, we studied in the production of a range of benzyl alchohol derivatives under the optimized parameter mention above (Table 2). As seen in Table, aryl aldehydes containing electron-donor and withdrawing groups effectively reducted by mesoporous TTSA@Ni. For example, reduction of aryl aldehydes containing $p$ - $\mathrm{Cl}, p-\mathrm{NO}_{2}, p-\mathrm{Me}, p-\mathrm{OMe}, p-\mathrm{N}(\mathrm{Me})_{2}, p-\mathrm{F}, p$ OH were successfully performed by mesoporous TTSA@Ni with excellent yields (2a-h). The transfer hydrogenation of aryl aldehydes with meta-substituted- $\mathrm{OH}$ and meta-substituted-F gave $90 \%$ and $84 \%$ yields, respectively $(2 \mathrm{i}, 2 \mathrm{j})$. The heterocycle aryl aldehydes such as furan-2-carbaldehyde, thiophene-2carbaldehyde and indole-3-carbaldehyde converted into corresponding benzyl alchohol with $88 \%, 90 \%$ and $93 \%$ reaction yields, respectively $(2 \mathrm{k}, 2 \mathrm{l}, 2 \mathrm{~m})$. It should be noted that transfer hydrogenation of terephthalaldehyde resulted in correspond 1,4-diol product in yields of $89 \%(2 n)$.

Catalytic studies revealed that mesoporous TTSA@Ni were successfully act as heterogeneous nanocatalyst in transfer hydrogenation of aryl ketones by giving with good to excellent reaction yields. The electron-rich groups such as $\mathrm{Me}, \mathrm{OMe}$, and $\mathrm{OH}$ at the para position of aryl ketones reacted smoothly by giving excellent reaction yields. For example, reduction of para-methoxy acethophenone, para-methyl acethophenone and para-hydroxy acethophenone resulted in yields of $92 \%, 88 \%$ and $80 \%$, respectively $(2 p, 2 m$ and $2 n)$. The electron-deficient groups such as $-\mathrm{Cl},-\mathrm{Br}$ at the para position of acetophenone converted efficiently to corresponding product by giving excellent reaction yields $96 \%$ and $86 \%$, respectively (2o, 2p). Other aryl ketone substrates such as 1-phenylbutane-1,3-dione (2r), 2-hydroxy-1,2diphenylethan-1-one (2s) and 1,2-bis(4-methoxyphenyl) ethane-1,2-dione (2t) were also investigated, the efficiency of forming the desired product was excellent.

\section{Proposed mechanism}

A proposed mechanism for synthesizing benzyl alcoholes was shown in Scheme 3. In the first stage, TTSA@Ni facilitates the decomposition of $\mathrm{HCOOH}$ in order to produce hydride species in the presence of 
triethyl amine. Finally, primary and secondary alchohol was obtained through hydrogenation ${ }^{2}$ (Scheme $3)$.

Lastly, reusability/recoverability of TTSA@Ni nanocomposite was studied on the reduction of benzaldehyde. First cycles were conducted in the presence of fresh TTSA@Ni nanocomposite. Following the catalytic tests, used catalyst was recollected from the reaction mixture via centrifusion and washed with water, dried and it was then directly used in consecutive catalytic runs. Reusability/recoverability tests displayed that TTSA@Ni nanocatalyst remained active even after six successive cycle $(98,96,94$, $93,88,84$ ) (Fig. 8).

\section{Experimental}

\section{Synthesis of silica@ triazine-thiourea-sulfonamide $\left(\mathrm{SiO}_{2} @ \mathrm{TTSA}\right)$ :}

A mixture of benzene-1,3-disulfonyl chloride ( $1 \mathrm{mmol}), 1,3,5$-triazine-2,4,6-triamine $(0.2 \mathrm{mmol})$, thiourea $(0.7 \mathrm{mmol})$ and silica nanoparticles $(0.05 \mathrm{~g})$ in $25 \mathrm{~mL}$ of dry acetonitrile under reflux condition was stirred for $12 \mathrm{~h}$. After the reaction was completed, the $\mathrm{SiO}_{2} @ T T S A$ were filtered off and washed with acetonitrile (weight: $120 \mathrm{~g}$ ) (Scheme 1).

\section{Synthesis of porous triazine-thiourea-sulfonamide (TTSA):}

In order to create porosity in the structure of $\mathrm{SiO}_{2} @ T$ TSA nanocomposite, the silica nanoparticles of the $\mathrm{SiO}_{2} @ T T S A$ nanocomposite was selectively removed through etching of the $\mathrm{SiO}_{2}$ nanoparticles. To $\mathrm{SiO}_{2} @$ TTSA nanocomposite $(0.5 \mathrm{~g}), \mathrm{HF}$ solution $(10 \mathrm{~mL}, 10 \mathrm{wt} \%)$, and deionized water $(10 \mathrm{~mL})$ was added and the mixture was stirred at room temperature for $4.5 \mathrm{~h}$. The porous TTSA was filtered off, washed well with water, and dried in air (weight: $0.3 \mathrm{~g}$ ) (Scheme 1).

\section{Nickel immobilization on the porous triazine-thiourea-sulfonamide (TTSA@Ni):}

$0.1 \mathrm{~g}$ of mesoporous TTSA and $\mathrm{NiCl}_{2}-6 \mathrm{H}_{2} \mathrm{O}(2 \mathrm{~mL}, 1 \mathrm{M})$ was refluxed for $3 \mathrm{~h}$. Then $12 \mathrm{mmol} \mathrm{NaBH}_{4}$ is dissolved in $10 \mathrm{~mL} \mathrm{H} \mathrm{H}_{2} \mathrm{O}$ was added to the reaction medium and stirred for $4 \mathrm{~h}$ at room temperature. After this period, the mesoporous TTSA@Ni were collected, rinsed with water:ethanole (1:1) and dried ${ }^{24}$ (Scheme 1).

\section{Preparation of benzyl alchohol derivatives using TTSA@Ni:}

In a $10 \mathrm{~mL}$ flask, a combination of ${\mathrm{HCOOH}-\mathrm{NEt}_{3}}_{3}(1: 0.7)$ was dissolved in water $(1 \mathrm{~mL})$ to prepare a solution with $\mathrm{pH}$ of 3.2. Then, the benzaldehyde or acetophenone derivatives $(1 \mathrm{mmol})$ and TTSA@Ni nanocomposites catalyst $(0.03 \mathrm{~g})$ were added to the reaction mixture and stirred at $80^{\circ} \mathrm{C}$. The development of the reaction was followed by TLC ( $n$-hexane/ethyl acetate, 10:3). After the completion of the reaction, mesoporous TTSA@Ni were separated via centrifusion and the reaction mixture was extracted EtOAc $(20 \mathrm{~mL})$. The solvent was evaporated to obtain benzyl alchohol derivatives (Scheme 2). 
In some products, the crude product was purified by column chromatography, eluting with hexane/EtOAc, to obtain the desired product (Scheme 2).

\section{Conclusion}

The main goal of the current study was to synthesis a novel mesoporous polysulfonamide-based catalyst via silica template technique. The results of this investigation show that the favorable surface properties, amphiphilicity and high nickel loading led to high catalyst performance for hydrogenation of aldehydes/ketones in aqueous solution. Moreover, it was found that mesoporous TTSA@Ni were reused six time without any significant loss of its activity.

\section{Declarations}

\section{Competing interest: No}

\section{References}

1. Nur, H., Ikeda, S. \& Ohtani, B. Phase-boundary catalysis of alkene epoxidation with aqueous hydrogen peroxide using amphiphilic zeolite particles loaded with titanium oxide. J. Catal. 204, 402-408 (2001).

2. Panahi, F., Haghighi, F. \& Khalafi-Nezhad, A. Reduction of Aldehydes with formic acid in ethanol using immobilized iridium nanoparticles on a triazine-phosphanimine polymeric organic support. Appl Organomet Chem. 34, e5880 (2020).

3. Mao, D. et al. Highly effective hybrid catalyst for the direct synthesis of dimethyl ether from syngas with magnesium oxide-modified HZSM-5 as a dehydration component. J. Catal. 230, 140-149 (2005).

4. Tejero, J. et al. Dehydration of 1-pentanol to di-n-pentyl ether over ion-exchange resin catalysts. J. Mol. Catal. Chem. 182, 541-554 (2002).

5. Manabe, K., limura, S., Xiang, M. S. \& Kobayashi, S. Dehydration reactions in water. brønsted acidsurfactant-combined catalyst for ester, ether, thioether, and dithioacetal formation in water. J. Am. Chem. Soc. 124, 11971-11978 (2002).

6. Maleki, A., Taheri-Ledari, R., Ghalavand, R. \& Firouzi-Haji, R. Palladium-decorated ophenylenediamine-functionalized $\mathrm{Fe}_{3} \mathrm{O}_{4} / \mathrm{SiO}_{2}$ magnetic nanoparticles: A promising solid-state catalytic system used for Suzuki-Miyaura coupling reactions, J. Phys. Chem. Solids, 136,109200109208(2020).

7. Mahmoodi, M., Bamoniri, A. \& Taherpour, A. A. One-pot synthesis of $2 \mathrm{H}$-indazolo[2,1-b]phthalazinetriones via nano $\mathrm{Y}$-Al203/BF3/Fe304 as an efficient catalyst and theoretical DFT study on them. $J$. Heterocycl. Chem. 57, 1-14 (2020). 
8. Hemmati, S., Heravi, M. M., Karmakar, B. \& Veisi, H. Green fabrication of reduced graphene oxide decorated with Ag nanoparticles (rGO/Ag NPs) nanocomposite: A reusable catalyst for the degradation of environmental pollutants in aqueous medium. J. Mol. Liq. 319, 114302-114311 (2020).

9. Ghasemi, P., Yarie, M., Zolfigol, M. A., Taherpour, A. A. \& Torabi, M. Ionically tagged magnetic nanoparticles with urea linkers: application for preparation of 2-aryl-quinoline-4-carboxylic acids via an anomeric-based oxidation mechanism. ACS Omega. 5, 3207-3217 (2020).

10. Salehzadeh, H., Nematollahi, D. \& Hajian, A. 1,2,4-Triazole-3-thiol-protected silver-nanoparticles as a platform for ECE electrochemical reaction. Electrochem. Commun. 82, 56-60 (2017).

11. Fu, Y. et al. Au nanoparticles decorated on activated coke via a facile preparation for efficient catalytic reduction of nitrophenols and azo dyes. Appl. Surf. Sci. 473, 578-588 (2019).

12. Zeynizadeh, B., Rahmani, S. \& Tizhoush, H. The immobilized Cu nanoparticles on magnetic montmorillonite(MMT@Fe304@Cu): as an efficient and reusable nanocatalyst for reduction and reductive-acetylation of nitroarenes with NaBH4. Polyhedron. 175, 114201-114210 (2020).

13. Veisi, H., Najafi, S. \& Hemmati, S. Pd(II)/Pd(0) anchored to magnetic nanoparticles (Fe304) modified with biguanidine-chitosan polymer as a novel nanocatalyst for Suzuki-Miyaura coupling reactions. Int. J. Biol. Macromol. 113, 186-194 (2018).

14. Le, X. et al. Fibrous nano-silica supported palladium nanoparticles: an efficient catalyst for the reduction of 4-nitrophenol and hydrodechlorination of 4-chlorophenol under mild conditions. Catal. Commun. 59, 21-25 (2015).

15. Elazab, H. A., Siamaki, A. R., Moussa, S., Gupton, B. F. \& El-Shall, M. S. Highly efficient and magnetically recyclable graphene-supported Pd/Fe304 nanoparticle catalysts for Suzuki and Heck cross-coupling reactions. Appl. Catal. A: Gen. 491, 58-69 (2015).

16. Veisi, H., Hamelian, M. \& Hemmati, S. Palladium anchored to SBA-15 functionalized with melaminepyridine groups as a novel and efficient heterogeneous nanocatalyst for Suzuki-Miyaura coupling reactions. J. Mol. Catal. A: Chem. 395, 25-33 (2014).

17. (a) Lei, Y., Zhang, M., Li, Q., Xia, Y., Leng, G. A porous polymer-based solid acid catalyst with excellent amphiphilicity: an active and environmentally friendly catalyst for the hydration of alkynes, Polymers 11, 2091-2033 (2019); (b) Lei, Y. et al. Palladium supported on an amphiphilic porous organic polymer: a highly efficient catalyst for aminocarbonylation reactions in water, Mater. Chem. Front. 1, $1541-1549$ (2017).

18. Ye, Q. et al. Superior lubricity and antiwear performances enabled by porous carbon nanospheres with different shell microstructures. ACS Sustain. Chem. Eng. 14, 12527-12535 (2019).

19. Kim, K., Yoon, G., Baek, S., Rho, J. \& Lee, H. Facile nanocasting of dielectric metasurfaces with sub$100 \mathrm{~nm}$ resolution. ACS Appl. Mater. Interfaces. 11, 26109-26115 (2019).

20. Tanaka, S. et al. Mesoporous iron oxide synthesized using poly (styrene-b-acrylic acid-b-ethylene glycol) block copolymer micelles as templates for colorimetric and electrochemical detection of Glucose. ACS Appl. Mater. Interfaces. 10, 1039-1049 (2018). 
21. Dajek, M., Kowalczyk, R. \& Boratynski, P. J. trans-1,2-Diaminocyclohexane-based sulfonamides as effective hydrogen-bonding organocatalysts for asymmetric michael-hemiacetalization reaction. Catal. Sci. Technol. 8, 4358-4363 (2018).

22. a) Alavinia, S., Ghorbani-Vaghei, R., Rakhtshah, J., Yousefi Seyf, J. \& Arabian I. A. Copper iodide nanoparticles-decorated porous polysulfonamide gel: As effective catalyst for decarboxylative synthesis of N-arylsulfonamides, Appl. Organomet. Chem. 34, e5449(2020), b) a) Alavinia, S., Ghorbani-Vaghei, R. Synthesis of 3-oxadiazole-substituted imidazo[1,2-a]pyridines by nickel immobolized on multifunctional amphiphilic porous polysulfonamide-melamine, New. J. Chem. 44, 13062 (2020).

23. Alavinia, S. et al. a) Ghorbani-Vaghei, R.,, 304@Si02@propyl-ANDSA: A new catalyst for the synthesis of tetrazoloquinazolines, Appl. Organomet. Chem. 32, e4038(2018), b) Ghorbani-Vaghei, R., Alavinia, S., Merati, Z., Izadkhah, V. MNPs@SiO 2 -Pr-AP: A new catalyst for the synthesis of 2-amino-4aryl thiazole derivatives, Appl. Organomet. Chem. 32, e4127 (2018), c) Alavinia, S., Ghorbani-Vaghei, R. Targeted development of hydrophilic porous polysulfonamide gels with catalytic activity, J. Phys. Chem. Solids. 146, 109573-109584 (2020), d) Fatehi, A., Ghorbani-Vaghei, R., Alavinia, S., Mahmoodi, J. Synthesis of quinazoline derivatives catalyzed by a new efficient reusable nanomagnetic catalyst supported with functionalized piperidinium benzene-1,3-disulfonate ionic liquid, ChemistrySelect. 5, 944-951 (2020), e) Hamidi Dastjerdi, F., Ghorbani-Vaghei, R., Alavinia, S. Copper iodide nanoparticles immobilized porous polysulfonamide: an effective nanocatalyst for synthesis of imidazo [1,2-a] pyridines, Catal. Letter, 150, 1-9 (2020), f) Shekarlab, N., GhorbaniVaghei, R., Alavinia, S. Preparation and characterization of copper/polysulfonamide complex immobilized on geraphene oxide as a novel catalyst for the synthesis of pyrimido[1,2a]benzimidazoles, Appl. Organomet. Chem. 34 e5918 (2020), g) Solgi, S., Ghorbani-Vaghei, R., Alavinia, S. Application of copper iodide nanoparticles immobolized porous polysulfonamide gel as an effective nanocatalyst for synthesis of aminoindolizines, J. Porous. Mater. 1-10 (2020).

24. Khan, S., Ghatak, A. \& Bhar, S. Reductive homocoupling of benzylic halides in aqueous medium using recyclable alumina-supported nickel nanoparticles. Tetrahedron Lett. 56, 2480-2487 (2015).

\section{Tables}

Table 1: Optimization of the reaction conditions. 


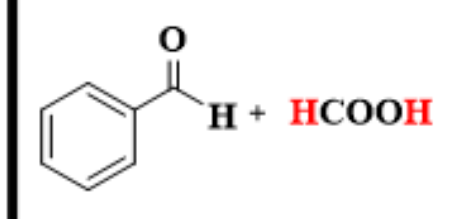

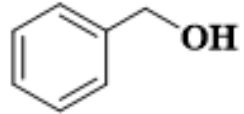

\begin{tabular}{|c|c|c|c|c|c|c|}
\hline Entry & $\begin{array}{c}\text { Cat. } \\
\text { (g) }\end{array}$ & Hydrogen doner & Solvent & $\begin{array}{c}\text { Temperature } \\
\left({ }^{\circ} \mathbf{C}\right)\end{array}$ & $\begin{array}{c}\text { Time } \\
\text { (h) }\end{array}$ & $\begin{array}{l}\text { Yield } \\
(\%)^{\mathrm{a}}\end{array}$ \\
\hline 1 & - & $\mathrm{HCOOH}$ & $\mathrm{H}_{2} \mathrm{O}$ & 80 & 24 & N.R. \\
\hline 2 & 0.03 & $\mathrm{HCOOH}$ & $\mathrm{H}_{2} \mathrm{O}$ & 80 & 24 & Trace \\
\hline 3 & 0.03 & $\mathrm{HCOONH}_{4}$ & $\mathrm{H}_{2} \mathrm{O}$ & 80 & 12 & 58 \\
\hline 4 & 0.03 & $\begin{array}{c}\mathrm{HCOOH}- \\
\mathrm{HCOONH}_{4} \\
(\mathrm{pH}=2.5)\end{array}$ & $\mathrm{H}_{2} \mathrm{O}$ & 80 & 6 & 80 \\
\hline 5 & 0.03 & $\begin{array}{c}\text { HCOOH-NEt } t_{3} \\
(1: 0.2, \mathrm{pH}=1.7)\end{array}$ & $\mathrm{H}_{2} \mathrm{O}$ & 80 & 6 & 40 \\
\hline 6 & 0.03 & $\begin{array}{l}\mathrm{HCOOH} \mathrm{NEt}_{3} \\
(1: 0.3, \mathrm{pH}=2.0)\end{array}$ & $\mathrm{H}_{2} \mathrm{O}$ & 80 & 6 & 60 \\
\hline 7 & 0.03 & $\begin{array}{c}\mathrm{HCOOH} / \mathrm{NEt}_{3} \\
(1: 0.5, \mathrm{pH}=2.5)\end{array}$ & $\mathrm{H}_{2} \mathrm{O}$ & 80 & 3 & 84 \\
\hline 8 & 0.03 & $\begin{array}{l}\text { HCOOH-NEt } \\
(1: 0.7, \mathrm{pH}=3.2)\end{array}$ & $\mathrm{H}_{2} \mathrm{O}$ & 80 & 0.5 & 98 \\
\hline 9 & 0.03 & $\begin{array}{c}\text { HCOOH-NEt } t_{3} \\
(1: 1, \mathrm{pH}=3.7)\end{array}$ & $\mathrm{H}_{2} \mathrm{O}$ & 80 & 0.5 & 95 \\
\hline 10 & 0.03 & $\begin{array}{l}\text { HCOOH-NEt } t_{3} \\
(1: 0.7, \mathrm{pH}=3.2)\end{array}$ & $\mathrm{H}_{2} \mathrm{O}$ & 80 & 0.5 & 95 \\
\hline 11 & 0.03 & $\begin{array}{l}\mathrm{HCOOH}-\mathrm{NEt}_{3} \\
(1: 0.7, \mathrm{pH}=3.2)\end{array}$ & $\mathrm{H}_{2} \mathrm{O}$ & R.T. & 12 & 45 \\
\hline 12 & 0.03 & $\begin{array}{l}\mathrm{HCOOH} \mathrm{NEt}_{3} \\
(1: 0.7, \mathrm{pH}=3.2)\end{array}$ & $\mathrm{EtOH}$ & 80 & 1 & 80 \\
\hline 13 & 0.03 & $\begin{array}{l}\text { HCOOH-NEt } t_{3} \\
(1: 0.7, \mathrm{pH}=3.2)\end{array}$ & $\mathrm{H}_{2} \mathrm{O}: \mathrm{EtOH}$ & 80 & 1 & 85 \\
\hline 14 & 0.03 & 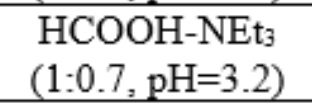 & THF & 80 & 24 & Trace \\
\hline 15 & 0.03 & $\begin{array}{l}\mathrm{HCOOH}-\mathrm{NEt}_{3} \\
(1: 0.7, \mathrm{pH}=3.2)\end{array}$ & $\mathrm{CH}_{3} \mathrm{CN}$ & 80 & 24 & 50 \\
\hline 16 & 0.03 & $\begin{array}{c}\mathrm{HCOOH}-\mathrm{NEt}_{3} \\
(1: 0.7, \mathrm{pH}=3.2)\end{array}$ & Solvent-free & 80 & 24 & 60 \\
\hline 17 & 0.05 & $\begin{array}{l}\mathrm{HCOOH} \mathrm{NEt}_{3} \\
(1: 0.7, \mathrm{pH}=3.2)\end{array}$ & $\mathrm{H}_{2} \mathrm{O}$ & 80 & 0.5 & 98 \\
\hline
\end{tabular}

${ }^{a}$ Isolated yield

Table 2: Reduction of different aldehydes and ketones to alcohols in the presence of TTSA@Ni nanocatalyst using formic acid-triethylamine as reducing agent. ${ }^{a}$ 


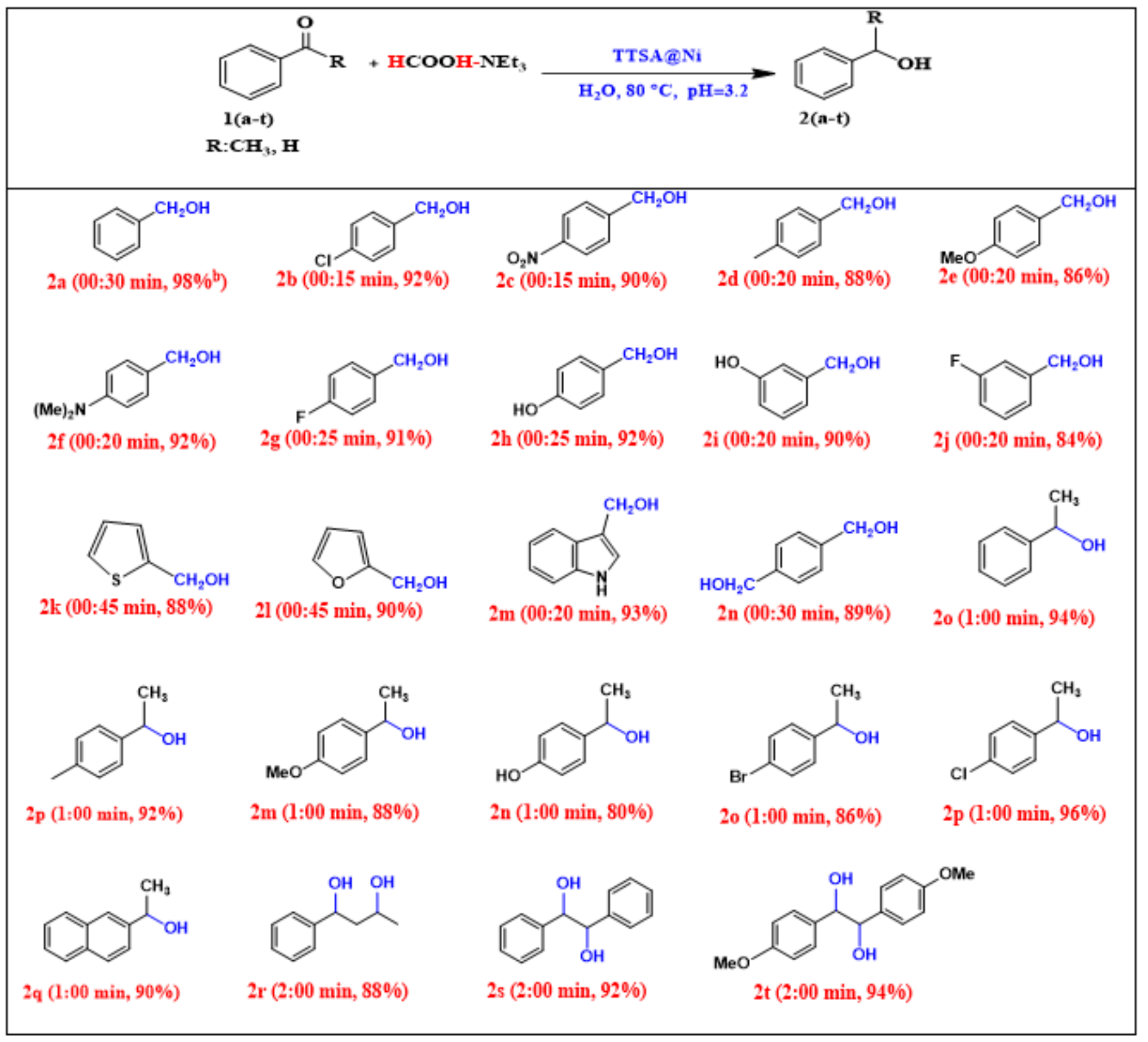

[a]Reaction condition: benzaldehyde or acetophenone derivatives (1 mmol), formic acid-triethyl amine (1: $0.7 \mathrm{mmol})$, TTSA@Ni (0.03 g), $\mathrm{H}_{2} \mathrm{O}(1 \mathrm{~mL}), 80^{\circ} \mathrm{C},{ }^{[b]}$ Isolated yield.

Figures 


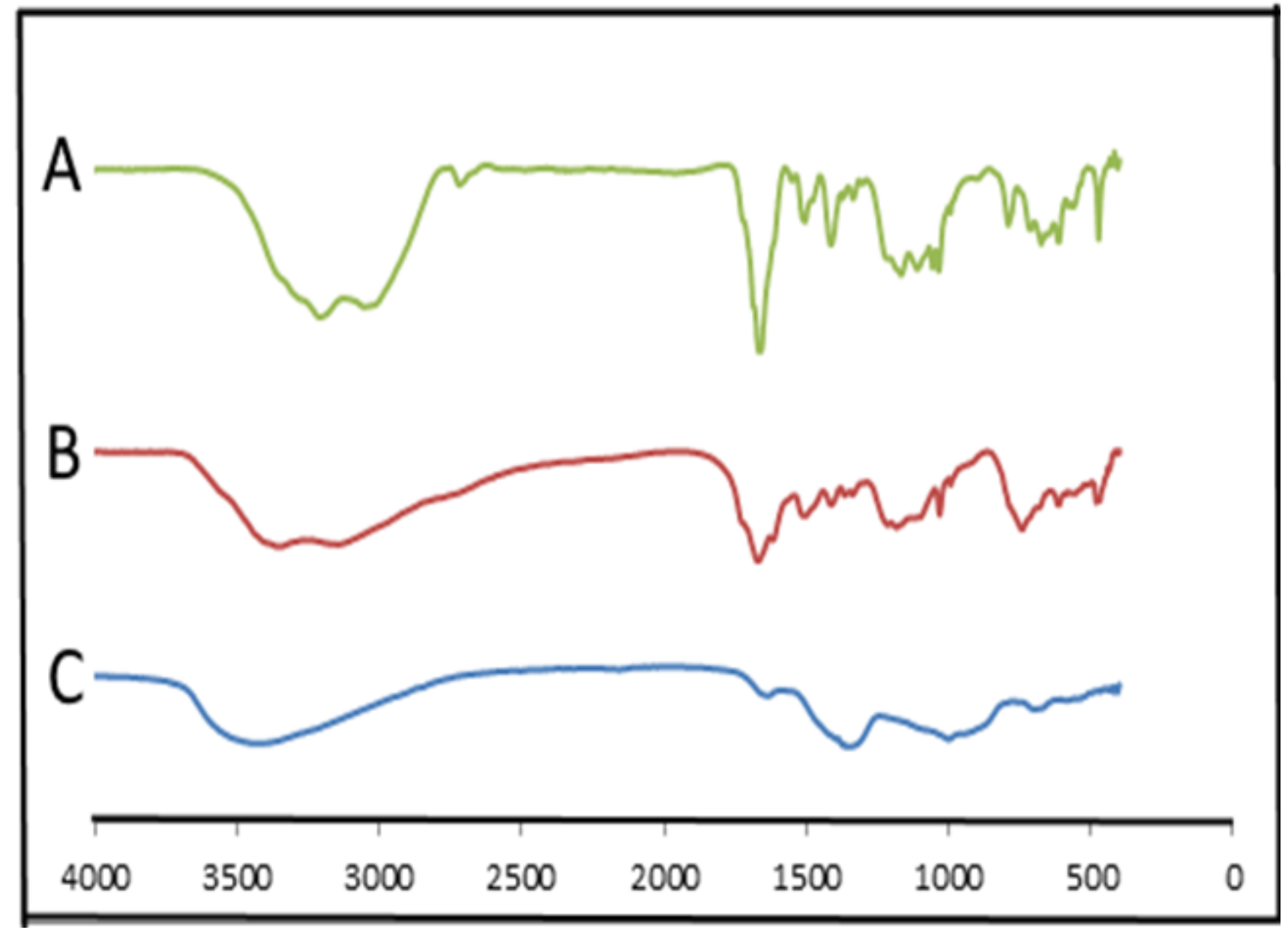

Figure 1

FT-IR spectrum of SiO2@TTSA NPs (A), Porous TTSA (B), TTSA@Ni nanocomposite (C).

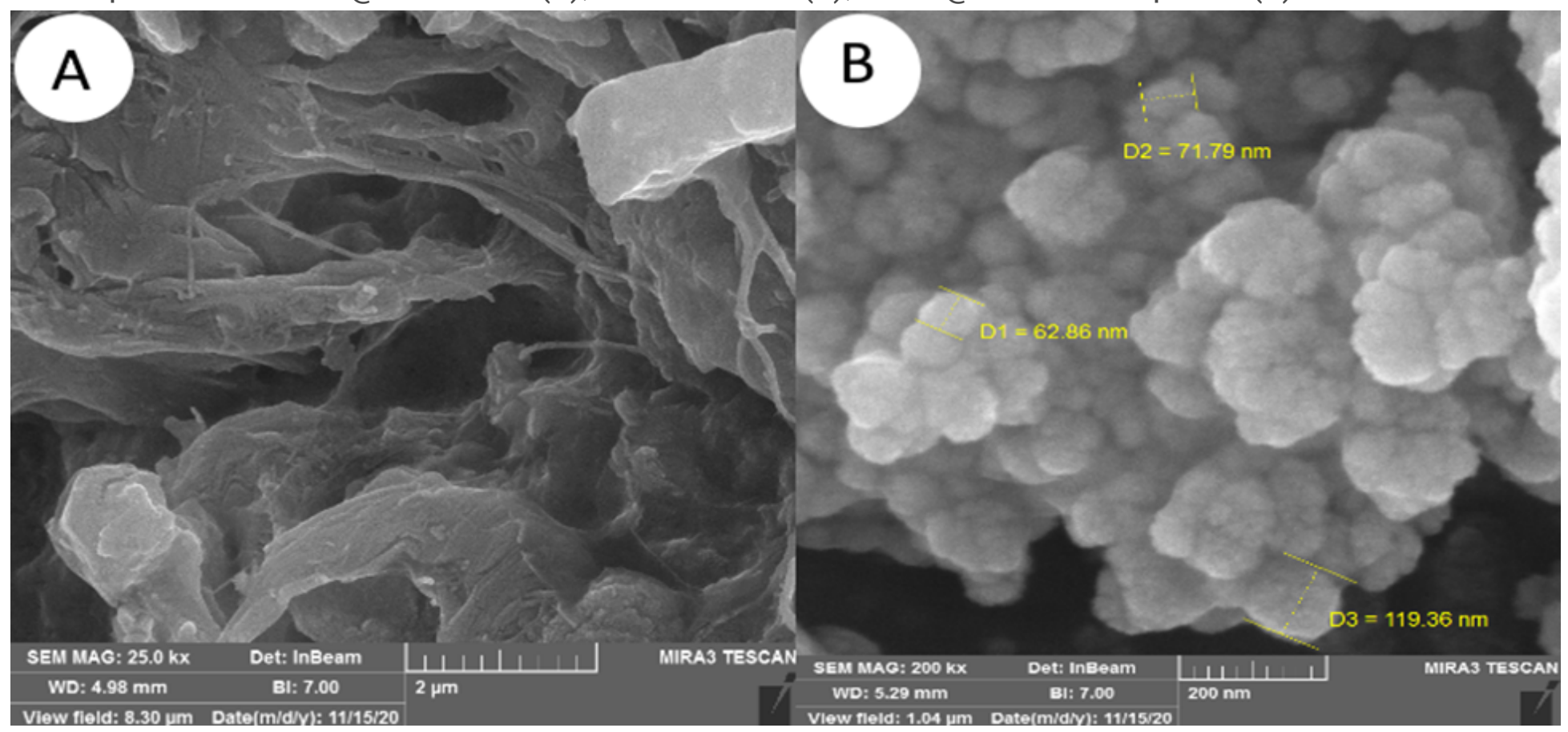

Figure 2 
FE-SEM photographs of porous TTSA (A) TTSA@Ni (B).

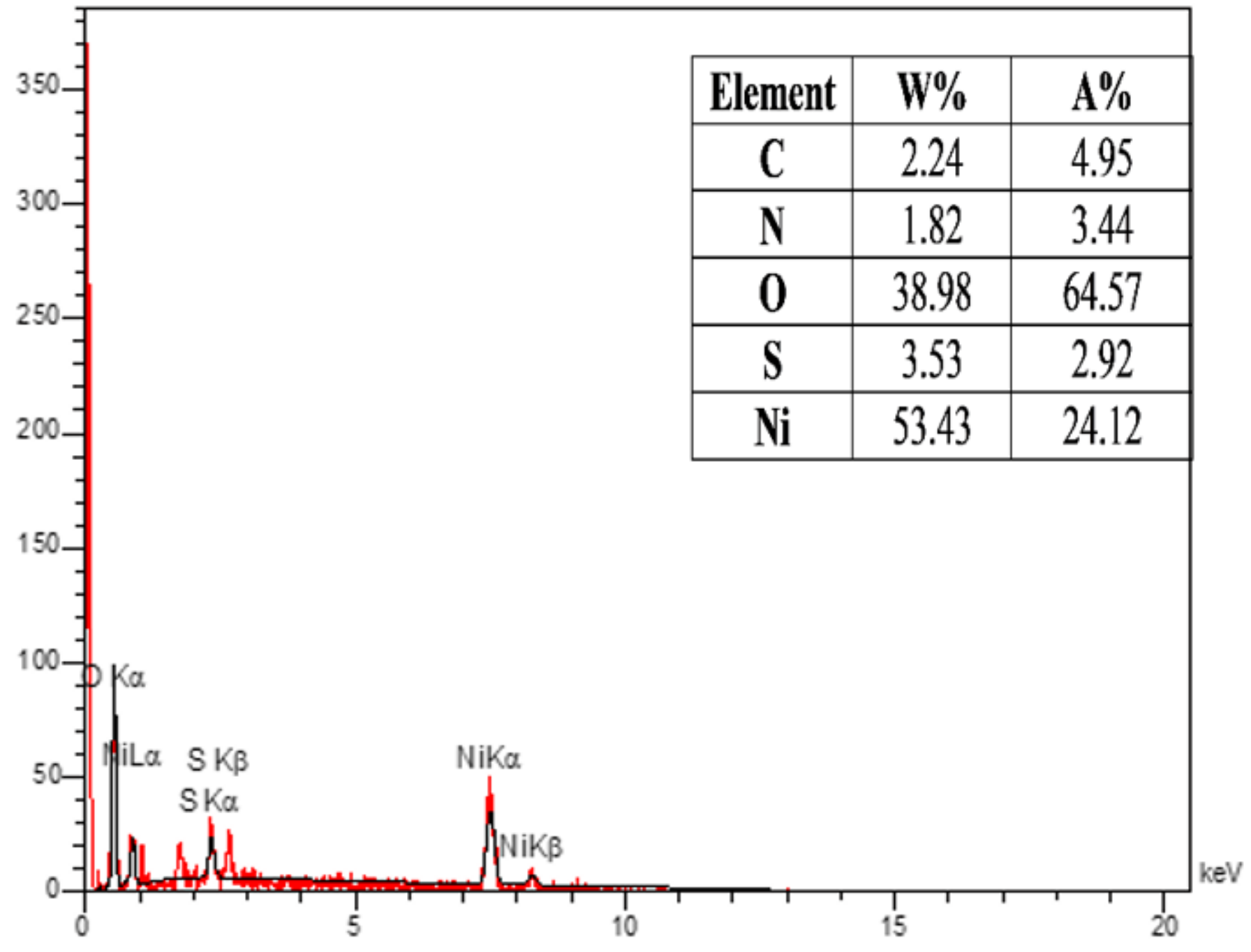

Figure 3

EDX spectrum of TTSA@Ni NPs. 


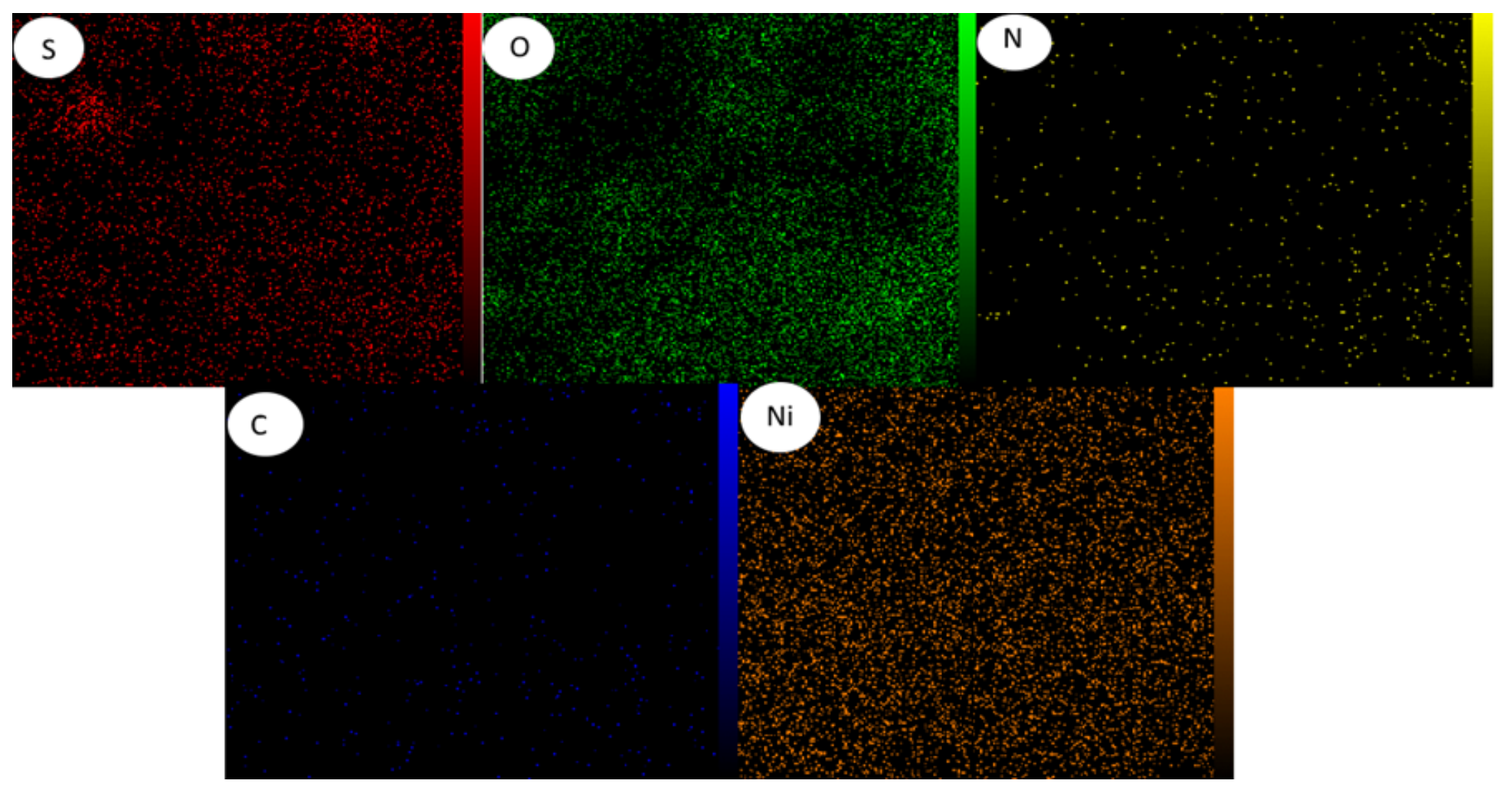

Figure 4

Elemental mapping of TTSA@Ni shows the presence of C, N, O, S, and $\mathrm{Ni}$ atoms in the nanocomposite. 


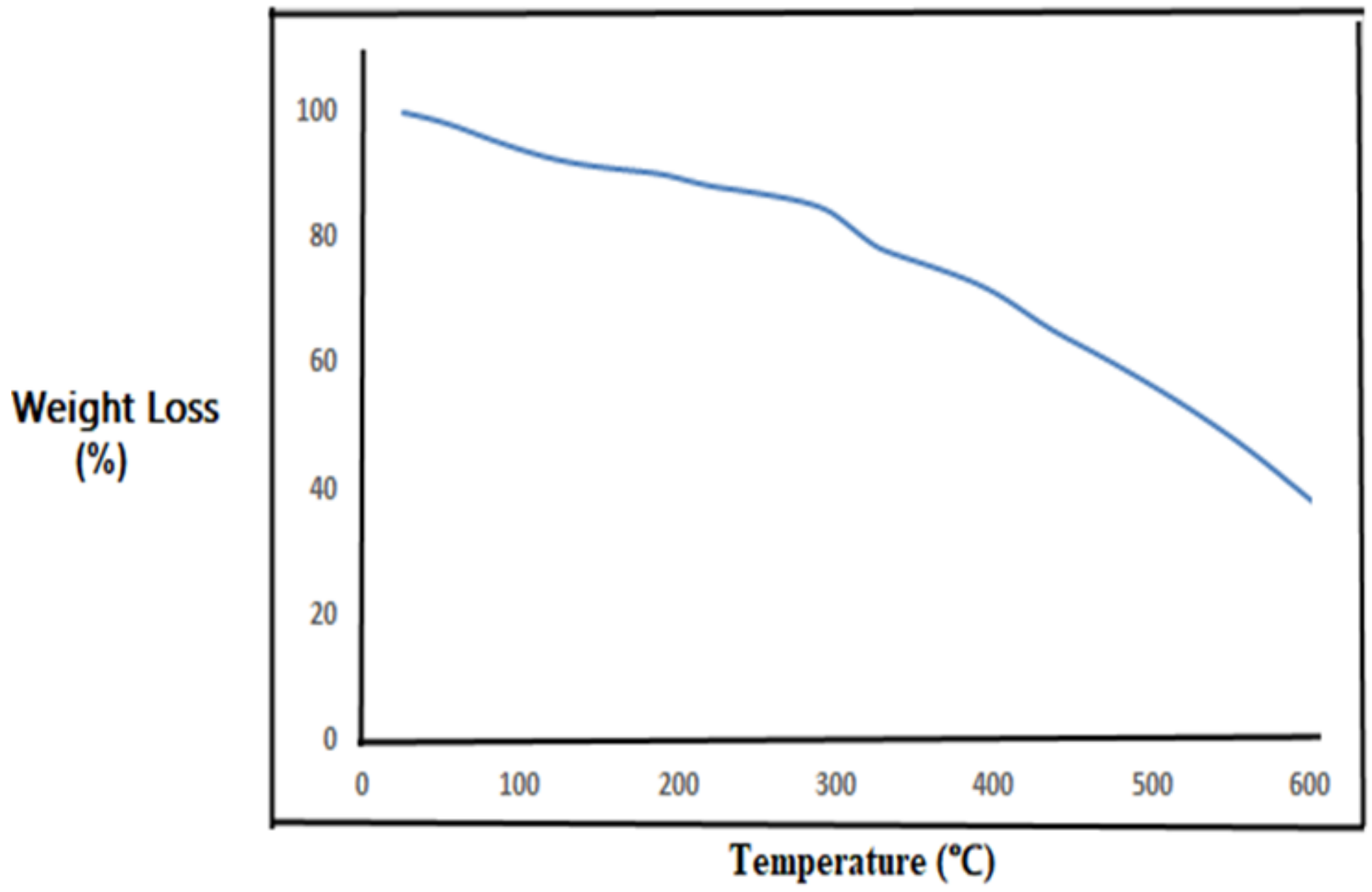

Figure 5

TGA curve of y-Fe203@talc@PBST NPs. 


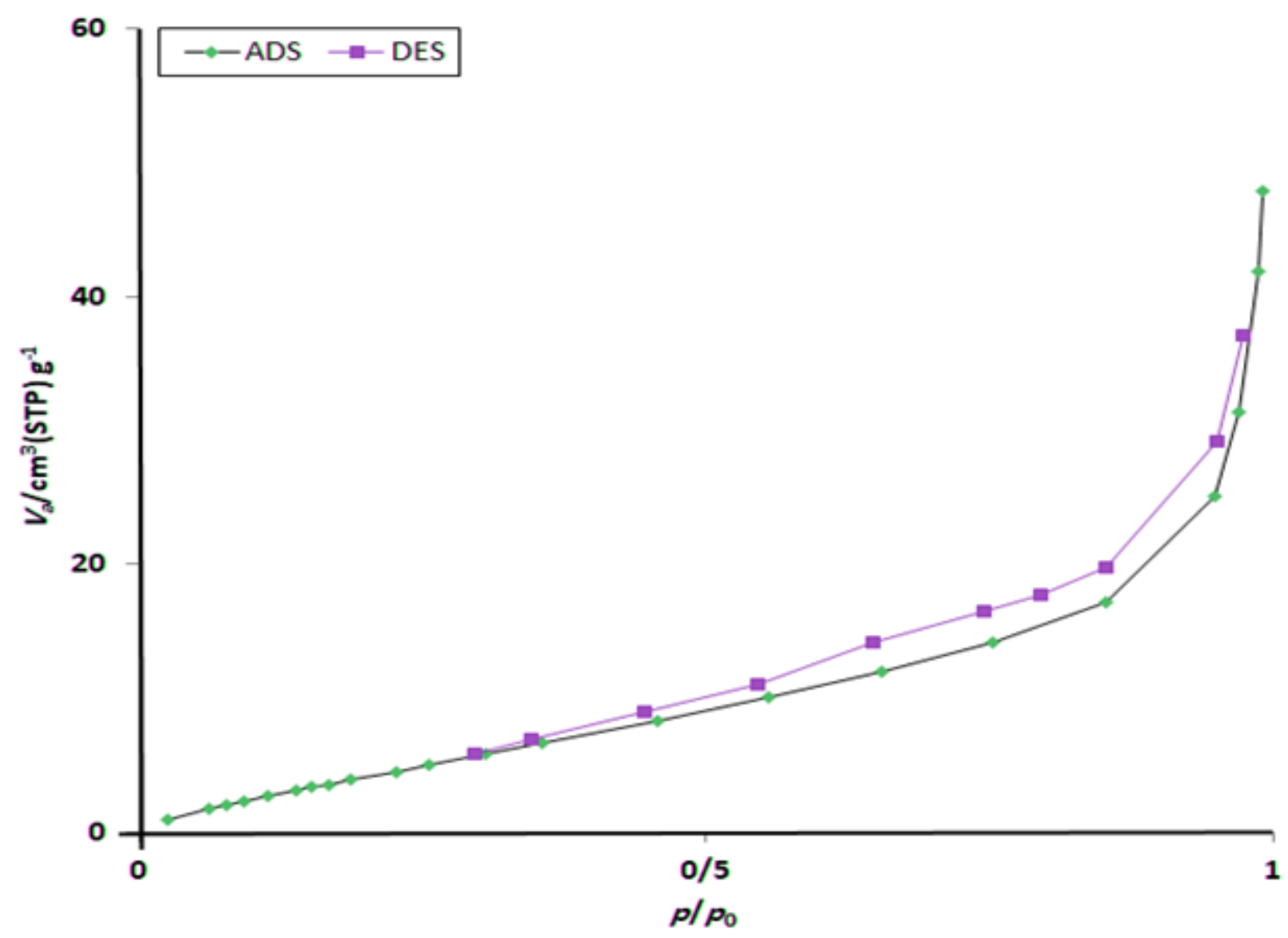

Figure 6

BET measurment of curve of TTSA polymer.

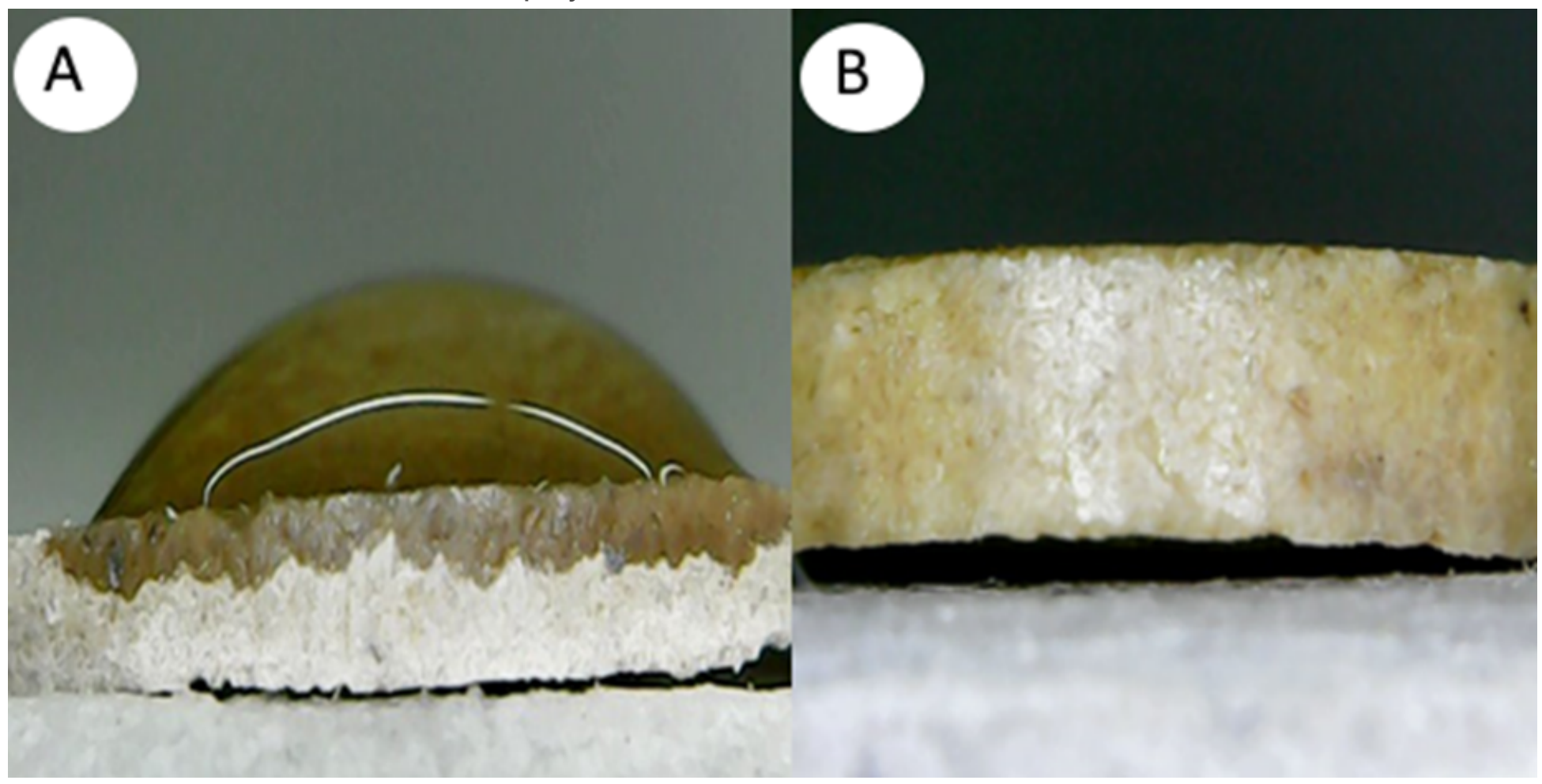

Figure 7 
Contact angles of TTSA@Ni, photograph of water (A) and oil droplet (B).

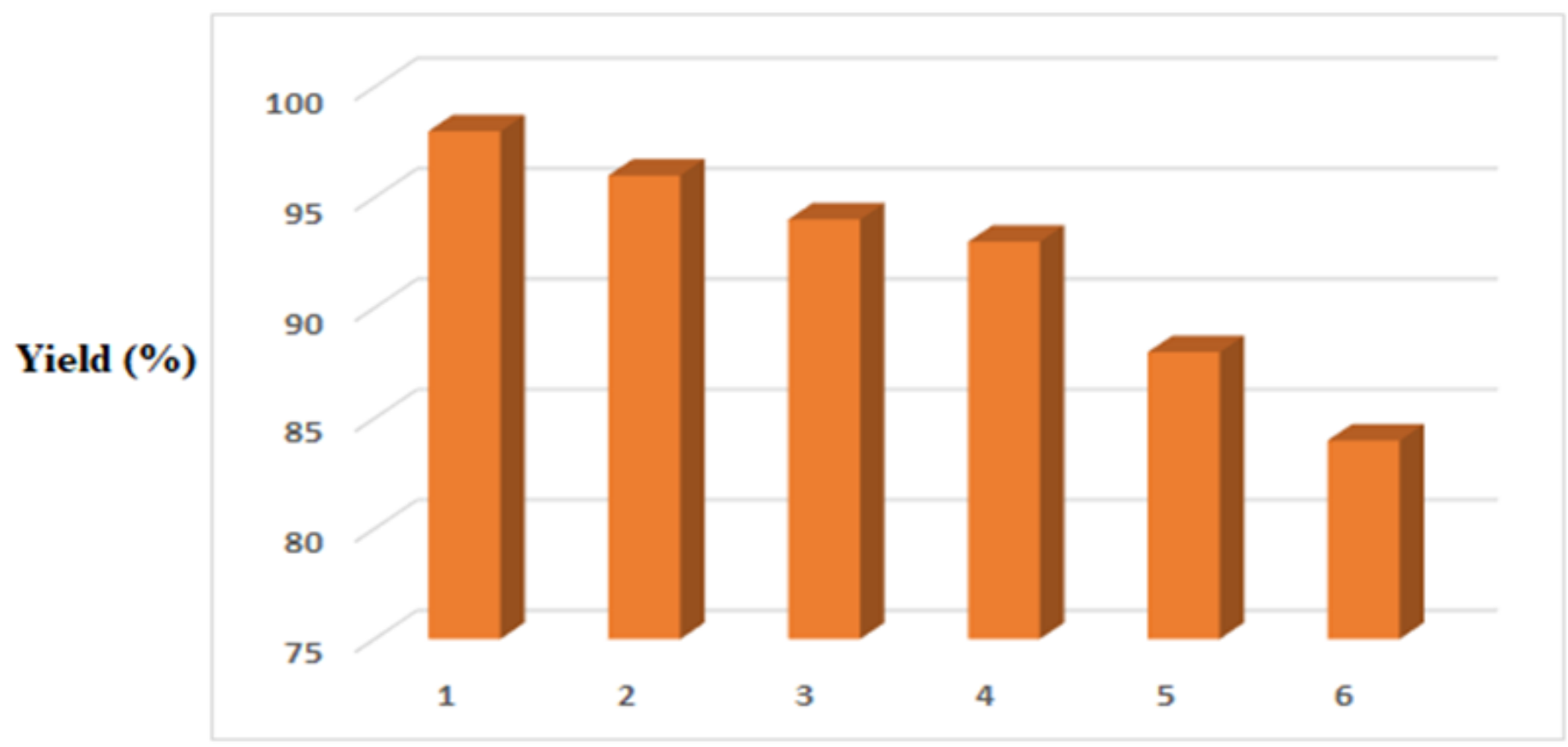

\section{Run}

Figure 8

Recycling of the TTSA@Ni for the reduction of benzaldehyde.

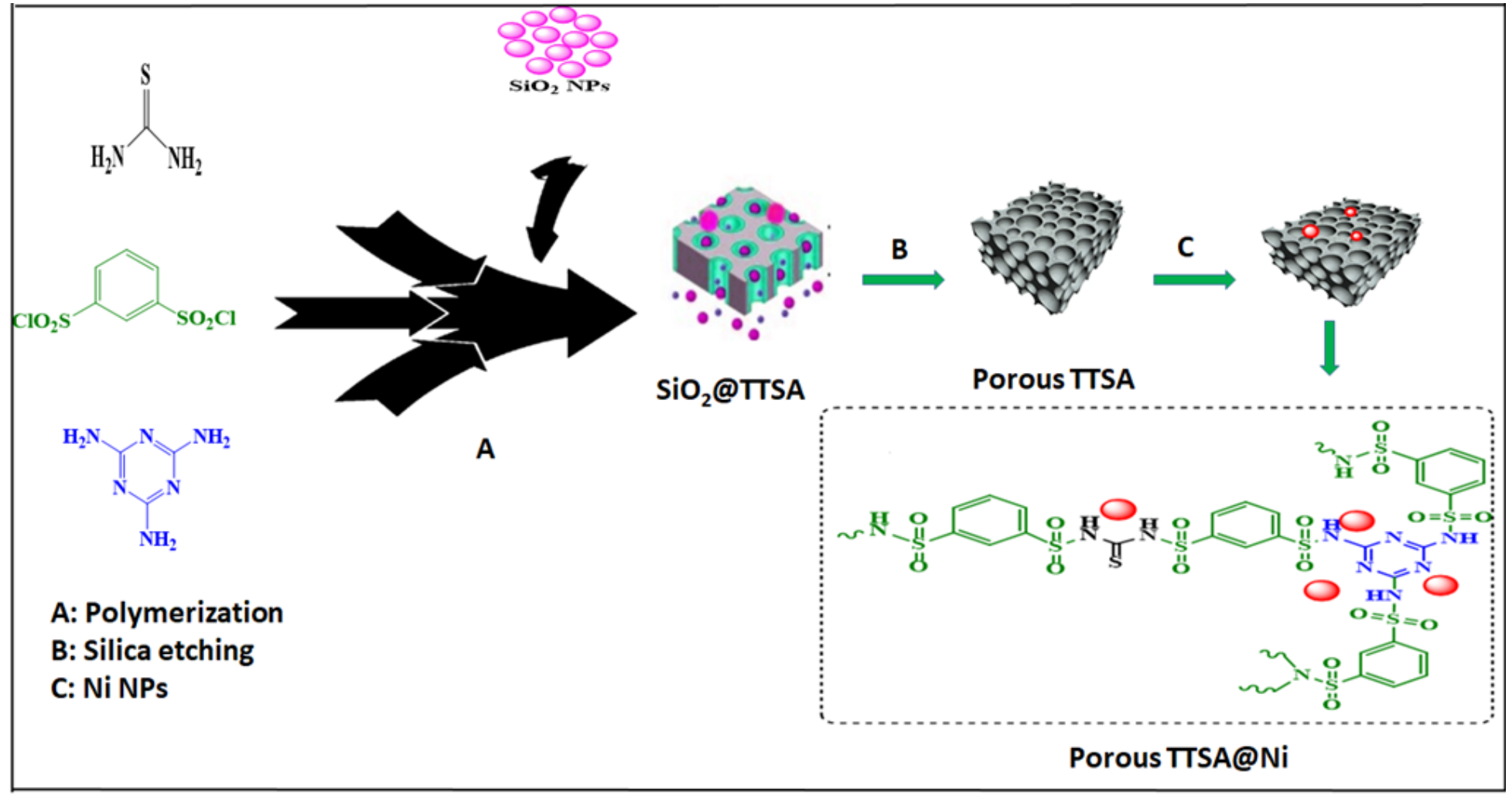

Figure 9 
The general route for the synthesis of mesoporousTTSA@Ni catalyst.<smiles>[R][X]c1ccc(C(C)=O)cc1</smiles>

$\mathrm{R}: \mathrm{H}, p-\mathrm{Me}, p$-OMe, $p$-Cl, $p$-NO $\mathrm{NO}_{2}, p$-F, $p$-N(Me) $)_{2, p}-\mathrm{OH}, m-\mathrm{OH}, m-\mathrm{F}$, Furan, Thiophene, Indole $\mathrm{R}^{\prime}: \mathrm{H}, p_{-} \mathrm{Me}, p_{-} \mathrm{OMe}, p_{-} \mathrm{Cl}, p-\mathrm{Br}, p_{-} \mathrm{OH}, \mathrm{Naphtaline}$

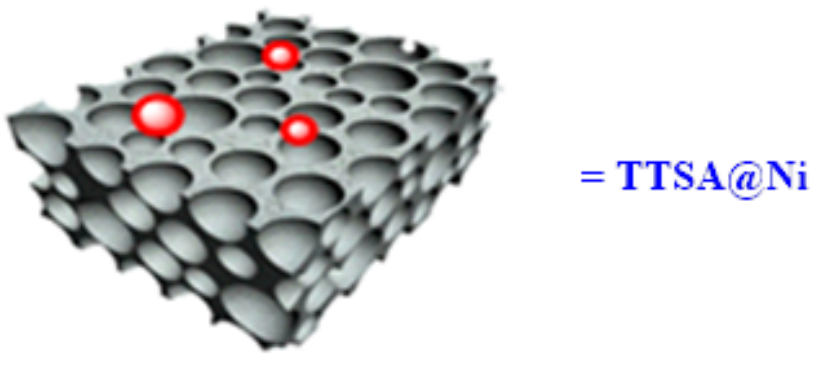

Figure 10

Transfer hydrogenation of aldehyde/ketone using mesoporous TTSA@Ni catalyst.

TTSA@Ni=

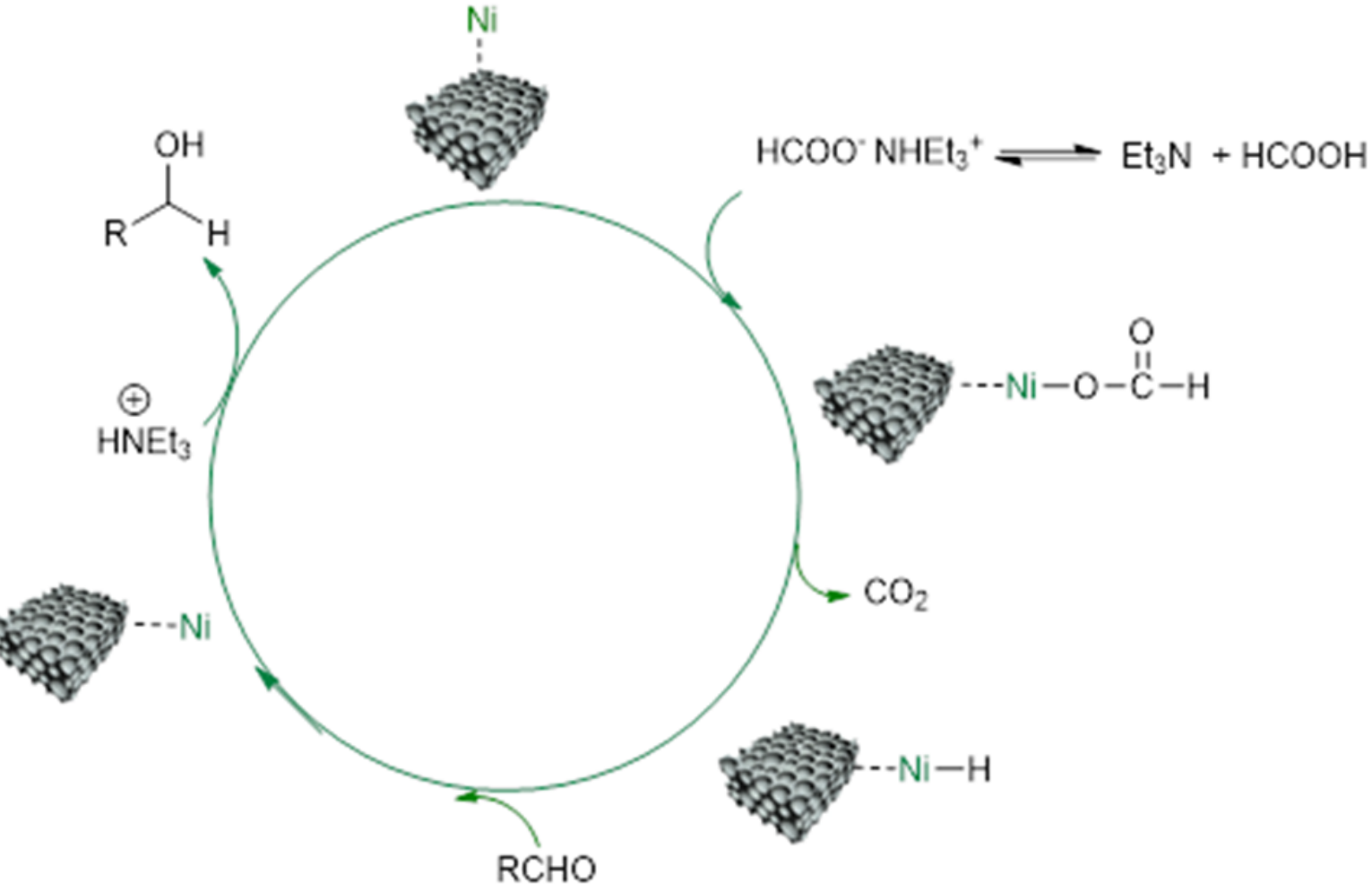


Figure 11

Proposed reaction mechanism for the reduction of carbonyl compounds using TTSA@Ni catalyst.

\section{Supplementary Files}

This is a list of supplementary files associated with this preprint. Click to download.

- SupportingInformation.docx 